The initial observations of spin-transfer torque were made in all-metal systems $^{5-9}$ - that is, with two ferromagnetic metal layers separated by a non-magnetic metal. Recently, the phenomenon has also been demonstrated in magnetic tunnel junctions ${ }^{10,11}$ (MTJs), in which the layer separating the two ferromagnetic layers is a thin insulator rather than a non-magnetic metal. In such a structure, when the magnetizations of the two ferromagnetic layers are parallel, the probability that electrons with similarly polarized spins will tunnel between them is high, and so its resistance to the flow of current is low. In contrast, when the magnetizations are antiparallel, the tunnelling probability is low and the resistance is high. The ability to distinguish the relative polarization of the two layers from their electrical resistance is one of the things that makes them attractive candidates for the basic building block of a magnetic random access memory, whose simple crosspoint architecture and non-volatile character considerably reduce the power consumption and increase the storage density. The free layer of such a structure could in principle be switched magnetically between the high and low resistance states (' 1 ' and ' 0 ' memory states), in the same way as the bits of a magnetic hard disk are switched. But doing so without affecting the magnetization of the underlying hard layer or those of neighbouring devices is a challenging task, and one that limits the miniaturization and ultimate storage density that could be achieved. Thankfully, the spin-transfer torque effect provides an electrically driven alternative. What has been missing has been accurate information about the details of this process, a shortcoming that the two present studies address ${ }^{3,4}$.
In the first study, Sankey and colleagues use a technique known as spin-transfer-driven ferromagnetic resonance (ST-FMR) to measure the bias- and angular-dependence of the spintransfer torque in MgO-based MTJs. In this technique the free magnetic moment of an MTJ is driven into resonance not by a microwave-frequency magnetic field, as occurs in conventional FMR, but by an electric current modulated at a microwave frequency. As this resonant dynamics is driven by the spin-transfer torque induced by the current bias, analysis of the ST-FMR signal enables the collection of detailed information on the magnitude and direction of this torque. At low currents, the authors find that the torque lies in the plane defined by the hard and free magnetizations of MTJ, and its magnitude is in excellent agreement with a prediction for highly spin-polarized tunnelling. With increasing bias, this inplane component remains large, which is quite unexpected in light of the decrease in tunnelling magnetoresistance that is observed. The authors also find that the torque vector rotates out of the plane, which may efficiently assist magnetic reversal in practical devices.

In the second study, Kubota and colleagues take a similar approach but exploit the so-called spin-torque diode effect to access quantitative information about the spin-transfer torque in MgO-based MTJs. This enables them to go a step further and extend the range of bias currents to the value where the spintransfer torque actually switches the free magnetic moment of an MTJ. Similar to Sankey et al., Kubota and colleagues find that the in-plane torque vector agrees well with predictions, and detect a considerable perpendicular component at higher biases. But, surprisingly, they find very different values for the in-plane torques necessary to promote the switching at positive and negative biases. The latter may indeed indicate the importance of the torque's perpendicular component for magnetic reversal.

The quantitative information on spin torque obtained by both groups ${ }^{3,4}$ has major implications for the use of nanoscale spin-transfer devices. First, they confirm the high efficiency of spin-transfer torque in MTJ-based magnetic memory operated at high biases. However, the threshold bias for magnetic switching is still too high for integration with existing electronic technology and too destructive for long-term operation using existing MTJ materials and structures. On this score, the results suggest that only marginal improvements in spin-transfer-torque efficiency will be possible, even if 100\% tunnelling spin-polarization were achieved. But they also suggest that the path to improving the other characteristics of MTJs for spin-torque applications could be achieved through optimizing other material properties such as magnetic anisotropy and damping. Whether this is the correct path to practical spintronic devices only time will tell.

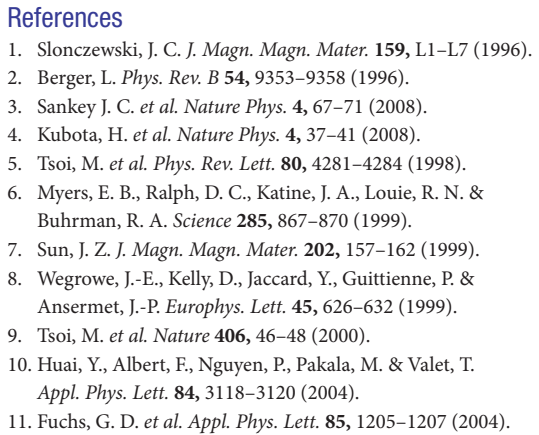

\title{
ERRATUM
}

\section{How to build a critical mind}

\author{
JOHN M. BEGGS
}

Nature Physics 3, 834-835 (2007)

In this News \& Views article, the second author name given in reference 6 was incorrect. The correct full reference is Levina, A., Herrmann, J. M. \& Geisel, T. Nature Phys. 3, 857-860 (2007). 